\title{
One-dimensional turbulence modeling of a turbulent counterflow flame with comparison to DNS
}

\author{
Z. Jozefik ${ }^{\mathrm{a}}$, A. R. Kerstein ${ }^{\mathrm{b}}$, H. Schmidt ${ }^{\mathrm{a}}$, S. Lyra ${ }^{\mathrm{c}}$, H. Kolla ${ }^{\mathrm{c}}$, J. H. Chen ${ }^{\mathrm{c}}$ \\ ${ }^{a}$ Brandenburg Technical University Cottbus-Senftenberg, Siemens-Halske-Ring 14, 03046 \\ Cottbus, Germany \\ ${ }^{b}$ Consultant, Danville, CA. USA \\ ${ }^{c}$ Combustion Research Facility, Sandia National Laboratories, Livermore, CA. USA
}

\begin{abstract}
The one-dimensional turbulence (ODT) model is applied to a reactant-to-product counterflow configuration and results are compared with DNS data. The model employed herein solves conservation equations for momentum, energy, and species on a one dimensional (1D) domain corresponding to the line spanning the domain between nozzle orifice centers. The effects of turbulent mixing are modeled via a stochastic process, while the Kolmogorov and reactive length and time scales are explicitly resolved and a detailed chemical kinetic mechanism is used. Comparisons between model and DNS results for spatial mean and root-meansquare (RMS) velocity, temperature, and major and minor species profiles are shown. The ODT approach shows qualitatively and quantitatively reasonable agreement with the DNS data. Scatter plots and statistics conditioned on temperature are also compared for heat release rate and all species. ODT is able to capture the range of results depicted by DNS. However, conditional statistics show signs of underignition.

Keywords: counterflow, turbulent flame, one-dimensional-turbulence model, numerical simulations
\end{abstract}

\footnotetext{
*Corresponding author: jozefik@tu-cottbus.de
} 


\section{Introduction}

Turbulence-chemistry interaction models that are based on fundamental principles are important in turbulent reacting flow simulations to improve combustion efficiency and to reduce emissions. The existence of a wide range of

5 length and time scales in high Reynolds number flows representative of practical applications and the number of chemical species involved in combustion of hydrocarbon fuels makes Direct Numerical Simulations (DNS) computationally intractable [1].

A key requirement for robust turbulent combustion modeling is that the

10 model must be able to access a sufficient portion of the chemical-state manifold [2]. PDF models are advantageous in this regard, but are subject to significant limitations because they do not resolve flame structure. Flamelet models provide such resolution, but they rely on low-dimensional chemical manifolds. Thus, neither of these leading approaches to turbulent combustion modeling is fully 15 satisfactory. Similar considerations apply to other commonly used approaches.

One-dimensional turbulence (ODT) resolves flame structure in 1D without compromising chemical-state accessibility, and achieves major cost reduction relative to DNS through reduced spatial dimensionality. ODT is a fully resolved, unsteady stochastic simulation model that emulates the Navier-Stokes turbulence. ODT has two key features. First, the properties of the flow reside on a one-dimensional domain. This 1D formulation allows full resolution of the interaction between large scales and molecular transport scales within computationally affordable simulations. Second, because vortical overturns cannot occur on a 1D domain, turbulent advection is represented using mapping events whose occurrences are governed by a random process. Unlike the Reynolds-averaged Navier-Stokes (RANS) model and large-eddy simulation (LES), which model the small scale phenomena and retain the $3 \mathrm{D}$ representation of the flow, ODT resolves all the scales of motion but models 3D turbulence. Hence ODT cannot capture geometrical effects and coherent flow structures, other than the so-called 
to determine the eddy frequency and eddy-size distribution, thereby providing a phenomenologically sound basis for driving turbulence.

As a stand-alone model, ODT has been used to simulate homogeneous turbulent non-reacting [3, 4, 5, 6, 7, 8] and reacting flows [9, 10, 11, 12, 13]. Notably, for non-premixed combustion ODT has provided fundamental insights concerning the spatio-temporal features of extinction-reignition [11] and yields overall agreement, in considerable detail, with state-space statistics obtained from DNS of temporally developing jet diffusion flames [13, 14].

For stand-alone modeling of turbulent flows using ODT, one must define the dominant direction of mean property variation. For complex flows which may not have a single dominant direction, ODT has been used as a sub-grid scale model in both RANS [10, 15] and LES [16] to provide closure for reacting scalars in combustion. An alternative multi-dimensional approach called ODTLES is discussed in [7, 17, 18].

Here, we conduct numerical studies of a highly turbulent counterflow flame as a benchmark for validating stand-alone ODT. By operating in a turbulent Reynolds number regime of relevance to practical systems such as gas turbines and internal combustion engines, counterflow flames retain the interaction of turbulence and chemistry of these environments 19], but additionally offer several advantages including: (a) the achievement of high Reynolds numbers without pilot flames, which is particularly advantageous from a modeling standpoint; (b) compactness of the domain by comparison with jet flames, with advantages from both a diagnostic and computational viewpoint; and (c) reduction or elimination of soot formation due to high strain rates and low residence times. For these reasons, the system is ideally suited to be used for computational model validation. Moreover, a premixed counterflow configuration is considered, providing the first detailed validation of ODT turbulence-chemistry interactions in turbulent premixed flames.

This paper is organized as follows. Section 2.1 summarizes the mathematical formulations. Section 2.2 gives a short overview of ODT. For further depth on ODT, the reader is referred to $[3,20,21]$. Section 2.3 introduces the ODT 
counterflow specific models. Section 3 describes the current counterflow configuration and boundary conditions. In the results, section 4 we compare model predictions to DNS data for mean and RMS velocity and species profiles. We compare mixing rates by looking at scalar dissipation rates and flame extinction/ignition characteristics by looking at the probability distribution of heat release rate conditioned on a chosen progress variable. To compare the range of results provided by DNS and ODT, scatter plots over temperature are shown. Statistics conditioned on temperature are compared for a more stringent test of model performance. Finally, a sensitivity study is carried out for the ODT input parameters.

\section{ODT}

\subsection{Mathematical Formulation}

We solve the set of variable density zero-Mach-number equations in one spatial dimension in a Lagrangian framework on an adaptive grid. In all equations, $x$ is the ODT line direction.

Following the formulation in [22], we begin by writing the continuity equation in integral form for a control volume $V$ that encloses the mixture mass. In Lagrangian formulation, the system boundary moves with the mass-average velocity so that in the direction of the ODT domain no mass crosses the control volume boundary via convective transport, only through diffusive transport. Since there is no mass source term, the Reynolds transport theorem is written for the continuity equation as

$$
\frac{d}{d t} \int_{V} \rho d V=0,
$$

where $\rho$ is the density. For uniform properties inside the control volume, and in ${ }_{85} 1 \mathrm{D}$, the equation reduces to

$$
\frac{d}{d t}(\rho d x)=0, \quad \Rightarrow \quad \rho d x=\text { constant }
$$


where $d x$ is a Lagrangian interval. This shows, that during a time advancement of the partial differential equations, the total mass in a given grid cell is constant. The balance equations for momentum, species mass fractions, and enthalpy are

$$
\begin{gathered}
\frac{d}{d t}\left(u_{i}\right)=\frac{1}{\rho} \frac{\partial}{\partial x}\left(\mu \frac{\partial u_{i}}{\partial x}\right)+\beta \delta_{i 1} \\
\frac{d}{d t}\left(Y_{s}\right)=\frac{\dot{\omega}_{s}}{\rho}-\frac{1}{\rho} \frac{\partial j_{s}}{\partial x}, \\
\frac{d}{d t}(h)=-\frac{1}{\rho} \frac{\partial q}{\partial x}
\end{gathered}
$$

with $s=1, \ldots, n_{s}$ and $n_{s}$ is the number of different species in the gas mixture.

90 Here, $\mu$ is the dynamic viscosity of the mixture, $u_{i}$ with $i \in\{1,2,3\}$ are the three ODT velocity components, $\beta$ is a pressure source term model discussed in section 2.3.1. $Y_{s}$ is the mass fraction of species $s, \dot{\omega}_{s}$ is the chemical source term of species $s, h$ is the enthalpy of the mixture, and $p$ the pressure. $j_{s}$ is the species diffusive flux given by

$$
j_{s}=-\rho D_{s}\left(\frac{\partial Y_{s}}{\partial x}+\frac{Y_{s}}{\bar{M}} \frac{\partial \bar{M}}{\partial x}\right),
$$

where $D_{s}$ is the diffusion coefficient of species $s$ and $\bar{M}$ is the mean molecular weight. $q$ is the heat flux given by

$$
q=-\lambda \frac{\partial T}{\partial x}+\sum_{s=1}^{n_{s}} h_{s} j_{s}
$$

where $h_{s}$ is the enthalpy of species $s$ including the heats of formation, $\lambda$ is the thermal conductivity and $T$ is the temperature. For the equation of state of a mixture of ideal gases we have

$$
p=\rho T \frac{R}{\bar{M}}
$$

with $R$ denoting the ideal gas constant. 
Time advancement of eqs. (3)- 50 is solved numerically using standard firstorder finite-difference discretization and is advanced at a diffusive CFL constraint. Spatial discretization is second order on a uniform grid and formally first order on the currently used non-uniform grid. An adaptive mesh approach is used, such that the merging and splitting of grid cells is performed in a manner that conserves fluxes of transported quantities: mass, momentum, and energy. The grid is adapted based on a nominally uniform distribution of grid points along the arc length of the (centered and scaled) velocity, heat release rate, and species profiles [22]. A minimum grid cell size of $8 \mu \mathrm{m}$ is used, which is sufficiently small that no significant differences in results are observed when using a minimum grid cell size of $4 \mu \mathrm{m}$. The ratio of Kolmogorov length to $8 \mu \mathrm{m}$ minimum cell size allowed is 2.35 . The minimum cell size allowed is set prior to the simulation and controlled during time advancement. If the minimum cell size criteria is violated, then mesh adaption is performed and cells are merged conserving mass, momentum, etc. The integration of the mean chemical source terms (used in the explicit time advancement) is performed with a high order implicit method using the most recent version of the CVODE code of the SUNDIALS package [23]. This eliminates chemical stiffness and allows advancement at a diffusive CFL. Thermodynamic and transport properties as well as reaction ${ }_{120}$ rates are calculated using the $\mathrm{C}++$ interface of the CANTERA software package 24]. In this study we use the hydrogen combustion mechanism proposed in [25], that contains 21 reactions and 9 species.

\subsection{Turbulence Model}

In ODT, the turbulent motions that accelerate mixing are modeled through a series of stochastic rearrangement events. These events may be interpreted as the model analog of individual turbulent eddies which are referred to as 'eddy events' or simply 'eddies'. Each eddy event interrupts the time advancement of other processes and an instantaneous transformation is applied to the property profiles over some spatial interval $\left(x_{0}, x_{0}+l\right)$, where $x_{0}$ represents the eddy 130 starting location and $l$ is the eddy length. 


\subsubsection{Eddy events}

The eddy event is central to the ODT modeling approach. It models the effects of a three-dimensional eddy using a 1D rearrangement. Eddy events are qualitatively similar to turbulence in that they have the effect of increasing gradients by redistributing the fluid elements along the 1D domain. Each eddy event consists of two mathematical operations. One is a triplet map representing the fluid displacements associated with a notional turbulent eddy and the other is a kernel transformation. The functional form chosen for the triplet map is the simplest of a class of mappings that satisfy the physical requirements of measure preservation, continuity, and scale locality over the eddy interval. The triplet map is conveniently represented by its inverse $f(x)$, such that the map moves fluid at location $f(x)$ to location $x$, where $f(x)$ is of the form [3]

$$
f\left(x ; x_{0}, l\right) \equiv x_{0}+\left\{\begin{array}{ll}
3\left(x-x_{0}\right) & \text { if } x_{0} \leq x \leq x_{0}+\frac{1}{3} l \\
2 l-3\left(x-x_{0}\right) & \text { if } x_{0}+\frac{1}{3} l \leq x \leq x_{0}+\frac{2}{3} l \\
3\left(x-x_{0}\right)-2 l & \text { if } x_{0}+\frac{2}{3} l \leq x \leq x_{0}+l \\
x-x_{0} & \text { otherwise }
\end{array}\right\} .
$$

This mapping takes a line segment $\left[x_{0}, x_{0}+l\right]$ shrinks it to a third of its original length, and then places three copies on the original domain. The middle copy is reversed, which ensures that property fields remain continuous and introduces the rotational folding effect of turbulent eddy motion. All quantities outside the $\left[x_{0}, x_{0}+l\right]$ interval are unaffected. The triplet map is augmented by a kernel transformation to implement pressure-induced energy redistribution among velocity components while obeying energy and momentum conservation laws. This enables the model to simulate the tendency of turbulent eddies to drive the flow toward isotropy. The kernel function used is that of the vector formulation of ODT in [4, 5].

\subsubsection{Eddy rate distribution}

The ODT velocity profiles evolve through the specification of the occurrences of eddy events. Conversely, the velocity profile supplies information that 
determines the size, location, and frequency of these events. The eddy selection process is stochastic and follows the variable density formulation of Ashurst and Kerstein [4, 26]. The local rate of an eddy is taken to be $\lambda\left(x_{0}, l\right)=1 / l^{2} \tau$, and the total rate of all eddies is $\Lambda=\iint \lambda\left(x_{0}, l\right) d x_{0} d l$. Hence, the joint PDF of eddy parameters $x_{0}$ and $l$ is $P\left(x_{0}, l\right)=\lambda\left(x_{0}, l\right) / \Lambda$. Eddy occurrences are sampled from a Poisson distribution with mean rate $\Lambda$, with $x_{0}$ and $l$ parameters sampled from $P\left(x_{0}, l\right)$. To restrict the occurrence of unphysically large eddies, the maximum eddy size allowed is an input parameter that is problem specific. Similar to dimensional relationships applied to fully developed turbulence, for eddy events in ODT, a relationship can be formulated between an eddy's size, its associated energy, and a time scale. The eddy time scale $\tau$ is used to specify the eddy acceptance probability, and is computed as

$$
\frac{1}{\tau}=C \sqrt{\frac{2 K_{0}}{\rho_{0} l^{3}}\left(E_{k i n}-Z E_{v p}-E_{p e}\right)},
$$

where $E_{k i n}$ is a measure of kinetic energy as in [4], $\rho_{0}=\int \rho K^{2}(x) d x$, and $K_{0}=\int K^{2}(x) d x$ where $K(x)$ is the kernel function as in [4]. $E_{v p}$ is a viscous penalty defined using scaling arguments as $E_{v p}=\frac{1}{2} \bar{\mu}^{2} / \bar{\rho} l$, where $\bar{\rho}$ and $\bar{\mu}$ are the average density and harmonically averaged [9] viscosity in the eddy region and $E_{p e}$ is a potential energy change defined in section 2.3.3. The adjustable model parameter $C$ represents turbulence intensity and $Z$ represents a viscous penalty factor. The evaluation of $\tau$ depends on the instantaneous flow state, so 175 eddy occurrences are responsive to unsteadiness resulting from transient forcing or statistical fluctuations inherent in the eddy-sampling process. The eddy occurrences thus depend on the effects of prior eddies and affect future eddy occurrences. These dependencies induce spatio-temporal correlations among eddy events, leading to a physically based representation of turbulence intermittency.

\subsection{Counterflow Submodels}

\subsubsection{Advection model}

As noted in section 1] stand-alone ODT represents flow advancement in the dominant direction of mean property variation and is applicable mainly to flows 
that have such a direction. In thin shear flows such as jets and mixing layers, this direction is transverse relative to the mean flow, and an ODT domain oriented in this direction can be validly formulated as a closed system provided that temporal or spatial (streamwise) advancement is implemented in accordance with the experimental or DNS configuration. In spatially developing cases, ODT can be viewed as representation of flow evolution along a Lagrangian line of sight that is advected downstream.

In a counterflow, mean property variation is primarily along the streamwise direction, so the ODT domain is the axial line. This line, viewed as a thin cylinder, is subject in a counterflow to inflow from the jet nozzles at the ends of the cylinder and corresponding net lateral outflow to conserve cylinder volume. Accordingly, an advection model needs to be introduced to transport incoming fluid from the nozzles towards the stagnation point and to expel mass from the ODT line as the fluid moves towards the stagnation point. The fluid expulsion required here by the counterflow configuration is a fundamental difference between the current and previous ODT formulations. The additional modeling that is needed introduces some further empiricism into ODT. The present study is in part intended to serve as an introduction and an initial validation of this additional modeling.

We displace cell faces with advection velocity $u_{1}^{A}(x)$, given by

$$
u_{1}^{A}(x)=u_{1}(x)+u_{1}^{D}(x)
$$

where $u_{1}(x)$ is the ODT online velocity component time advanced in Eq. (33) and $u_{1}^{D}(x)$ is the dilatation velocity introduced in section 2.3 .2 and given by Eq. (15). The advecting velocity is thus the sum of two contributions, one that is unrelated to density changes and one that is caused by density changes. The total ODT axial advection then consists of eddy events (maps) and $u_{1}^{A}(x)$.

To model the effect of the velocity decelerating towards the stagnation point, ${ }_{210}$ a pressure source term, $\beta$, in Eq. (3) is prescribed by 


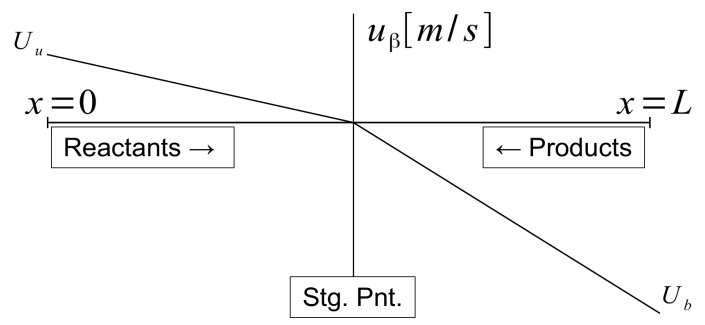

Figure 1: Shape function for pressure source term with prescribed stagnation point location. $U_{u}$ and $U_{b}$ are the mean inlet velocities of the reactants and products respectively.

$$
\beta(x)=u_{1} \cdot \frac{\partial u_{\beta}}{\partial x}
$$

The shape function for $u_{\beta}$ is shown in Fig. 1] where our simplified model linearly decelerates the incoming velocity from the nozzles towards the prescribed stagnation point. The stagnation point location is an empirical input parameter.

\subsubsection{Dilatation model}

In ODT we are living on a 1D line between the two nozzles. There is expansion/contraction occurring due to temperature-induced density changes. The pressure remains constant and therefore we must make a decision about how much dilatation to keep on our 1D line or inversely how much to expel. Starting from the continuity equation in Lagrangian form, we identify the preliminary dilatation velocity $\hat{u}_{i}^{D}$ using

$$
\frac{D \rho}{D t}+\rho \frac{\partial \hat{u}_{i}^{D}}{\partial x_{i}}=0
$$

in which the $x, y$ and $z$ directions correspond to the respective indices $i \in$ $\{1,2,3\}$. Solving for the preliminary dilatation velocity $\hat{u}_{1}^{D}$ in $x$

$$
\hat{u}_{1}^{D}(x)=-\int_{0}^{x} \alpha \frac{1}{\rho} \frac{D \rho}{D t} d x,
$$

where $\alpha=1 / 3$ is the fraction of the added volume that is kept on the line. For alpha, $1 / 3$ is chosen based on the reasoning that in a turbulent field, on average ${ }_{225} 1 / 3$ of the added volume is in the $x$ direction. For the final dilatation velocity $u_{1}^{D}$, 


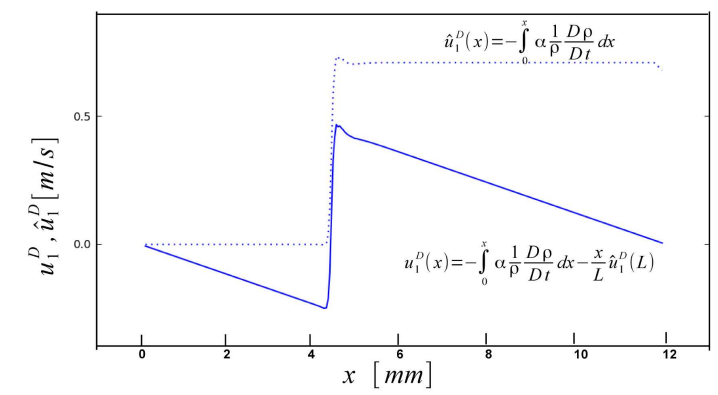

Figure 2: Preliminary and final dilatation velocity profiles for a notional laminar flow.

the boundary condition constraint dictated by the counterflow configuration, $u_{1}^{D}(0)=u_{1}^{D}(L)=0$ at the inlets, is imposed by linearly redistributing the total preliminary dilatation

$$
u_{1}^{D}(x)=-\int_{0}^{x} \alpha \frac{1}{\rho} \frac{D \rho}{D t} d x-\frac{x}{L} \cdot \hat{u}_{1}^{D}(L) .
$$

Fig. 2 shows schematically the preliminary and final dilatation velocity profiles over the domain for laminar flow.

\subsubsection{Darrieus-Landau instability model}

Planar flames are intrinsically unstable due to acceleration of the variabledensity fluid caused by thermal expansion across the burning front. This is the Darrieus-Landau instability, analogous to the Rayleigh-Taylor instability that develops when heavy fluid is above light fluid in a gravitational field. This analogy allows an existing ODT representation of the Rayleigh-Taylor instability [27] to be modified in order to incorporate the Darrieus-Landau instability mechanism into ODT. Namely, a formal analog of gravitational potential energy is introduced. It is based on the equivalence of downward (negative) gravity and upward (positive) acceleration. In our case, this implies that the constant acceleration of gravity is replaced by the varying time rate of change of the advecting velocity $u_{1}^{A}(x)$, defined as $a(x)=\partial u_{1}^{A}(x) / \partial t$. Based on [27], it then follows that the associated potential energy change resulting from triplet mapping the accelerating variable-density flow is 


$$
E_{p e}=\frac{8}{27} \int_{x_{0}}^{x_{0}+l} a(x) K(x)(\rho(f(x))-\bar{\rho}) d x,
$$

where the factor $8 / 27$ arises due to the variable density formulation and $\bar{\rho}$ is a reference density defined as the average density over the interval $\left[x_{0}, x_{0}+l\right]$. This potential energy change is nonzero only where the density varies, as it is the interaction of the dilatation-induced pressure gradient and the density gradient that is the cause of this instability mechanism. $E_{p e}$ is not a potential energy change in the same sense as in a buoyant flow, because it is not based on an external energy source. For this reason, it is only used to affect the probability of acceptance of an eddy, but it does not change the total kinetic energy during the energy redistribution step of the eddy event. It is however, a formal analog to the treatment of energy in the buoyant flow, and therefore a tunable coefficient 255 is not required. Reflecting the analogy to gravitational potential energy, $E_{p e}$ is subtracted from the available kinetic energy when computing eddy likelihood.

The Darrieus-Landau instability is not specific to the counterflow configuration nor is it inherently a finite-Mach-number effect, so a representation of the instability should be incorporated into any ODT formulation involving unsteady dilatation within the ODT domain, irrespective of Mach number. The DarrieusLandau model was first introduced in [28] and shown to provide quantitatively good results for the simulation of ignition times in a turbulent homogeneouscharged compression-ignition (HCCI). It has also been used in [29] and shown to improve results for modeling flame propagation in fuel beds of wildland fires.

265 In the Appendix, it is discussed further in the context of ODT modeling of flow acceleration effects.

\section{Counterflow Configuration}

A reactant-to-product counterflow configuration is investigated, which consists of two axisymmetric, opposed nozzles of internal diameter $D=12.7 \mathrm{~mm}$ separated by a distance $L_{x}=12.0 \mathrm{~mm}$ as shown in Fig. 3. The flow arrangement consists of a turbulent stream of premixed reactants supplied through the 
left nozzle at a volumetric flow rate of $Q_{u}=110 \mathrm{LPM}$ at an inlet temperature of $T_{u}=294 \mathrm{~K}$, and a laminar stream of hot combustion products in equilibrium at $T_{b}=1,475 \mathrm{~K}$ supplied through the right nozzle. The volumetric flow rate of the reactants fueling the stoichiometric flame, measured at $294 \mathrm{~K}$, is $Q_{b}=85 \mathrm{LPM}$. The thermochemical state of the product stream is obtained by taking the products of a stoichiometric hydrogen and air mixture with adiabatic flame temperature $2,388 \mathrm{~K}$ and cooling it under constant pressure to $1,475 \mathrm{~K}$. The reactant stream is shielded from the ambient air by an annular co-flow of nitrogen, supplied at $85 \mathrm{LPM}$. Combustion of a lean mixture of hydrogen and air ( $79 \% \mathrm{~N}_{2}$ and $21 \% \mathrm{O}_{2}$ by volume) at an equivalence ratio of $\phi_{u}=0.4$ and adiabatic flame temperature of $1,723 \mathrm{~K}$ at $101.3 \mathrm{kPa}$ flowing against a hot stream of combustion products generated by a stoichiometric flame with adiabatic flame temperature 2,388 $\mathrm{K}$ is established at an elevated turbulent Reynolds number and bulk strain rate in a compact cylindrical volume and away from solid boundaries. At the simulated conditions, the freely propagating laminar flame speed and nominal thickness of a one-dimensional flame are $S_{L}^{0}=0.22 \mathrm{~m} / \mathrm{s}$ and $L_{F}=\alpha / S_{L}^{0}=0.141 \mathrm{~mm}$, respectively, where $\alpha$ is the thermal diffusivity of the unburnt mixture. The laminar flame time is $t_{L}=L_{F} / S_{L}^{0}=0.64 \mathrm{~ms}$.

The bulk velocities of the two streams are computed from the volumetric flow rate and the nozzle diameter. Under these conditions the mean bulk strain rate is $a=2 \cdot U_{u} / L_{x}=2,400 \mathrm{~s}^{-1}$ and the residence time is $\tau_{R}=0.5 \cdot L_{x} / U_{u}=$ $0.4 \mathrm{~ms}$. The reactant side inlet turbulence is characterized by turbulence intensity, $u^{\prime} / U_{u}=0.35$, and an integral length scale, $L_{11} / D=0.30$, resulting in an eddy turn-over time, $t_{e}=L_{11} / u^{\prime}=0.752 \mathrm{~ms}$. The physical and numerical parameters of the simulations are summarized in Table 1

\subsection{ODT set-up}

The ODT domain spans the 1D domain between nozzle orifice centers, $L_{x}=12 \mathrm{~mm}$. To produce the reactant side turbulent inlet conditions, velocity fluctuations are superimposed on the mean inlet velocity at the reactants stream inlet. These fluctuations are obtained from a homogeneous isotropic turbulence 
Table 1: Numerical and physical parameters

$\begin{array}{lr}\text { Jet diameter }(\mathrm{D}) & 12.7 \mathrm{~mm} \\ \text { Mean inlet velocity of reactants }\left(U_{u}\right) & 14.47 \mathrm{~m} / \mathrm{s} \\ \text { Mean inlet velocity of products }\left(U_{b}\right) & 49.97 \mathrm{~m} / \mathrm{s} \\ \text { Temperature of reactants }\left(T_{u}\right) & 294 \mathrm{~K} \\ \text { Temperature of products }\left(T_{b}\right) & 1,475 \mathrm{~K} \\ \text { Turbulence intensity }\left(u^{\prime} / U_{u}\right) & 0.35 \\ \text { Integral length scale }\left(L_{11} / D\right) & 0.30 \\ \text { Bulk strain rate }\left(a=2 U_{u} / L_{x}\right) & 2,400 \mathrm{~s}^{-1} \\ \text { Jet Reynolds number }\left(R e_{j e t}=U_{u} D / \nu_{u}\right) & 10,400 \\ \text { Turbulent Reynolds number }\left(R e_{t}=u^{\prime} L_{11} / \nu\right) & 1,100 \\ \text { Karlovitz number }\left(K a=\left(L_{11} / L_{F}\right)^{-1 / 2}\left(u^{\prime} / S_{L}^{0}\right)^{3 / 2}\right) & 26 \\ \text { Damköhler number }\left(D a=R e_{t}^{1 / 2} / K a\right) & 1.2 \\ \text { Kolmogorov length scale }\left(\eta / D=R e^{-3 / 4} L_{11} / D\right) & 0.00157 \\ \text { DNS domain size }\left(L_{x} \times L_{y} \times L_{z}\right) & 432 \times 640 \times 640 \\ \text { DNS grid points }\left(N_{x} \times N_{y} \times N_{z}\right) & 12.0 \mathrm{~mm} \\ \text { ODT domain length }\left(L_{x}\right) & 0.95 D \times 1.48 D \times 1.48 D\end{array}$




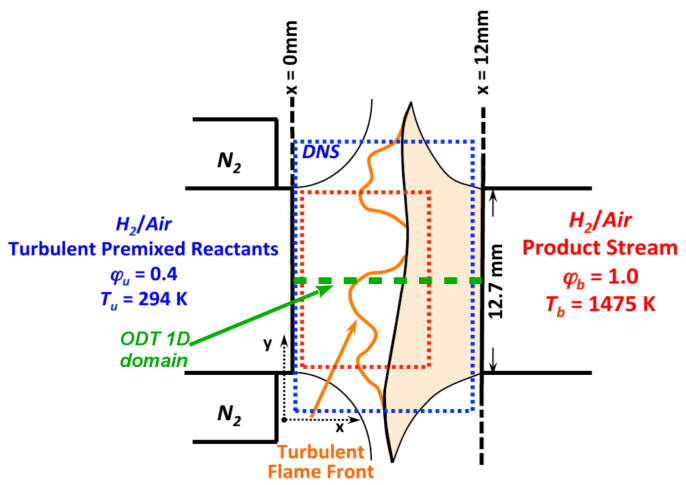

Figure 3: Schematic representation of the investigated counterflow burner. The ODT domain, denoted by the green dashed line, spans the 1D domain between nozzle orifice centers. The DNS data were obtained in a $12 \times 17.5 \times 17.5 \mathrm{~mm}^{3}$ rectangular volume denoted by the blue dotted box in the spanwise mid-plane. The DNS diagnostics window in which the analysis of the data is performed is confined to the $8.4 \times 8 \times 8 \mathrm{~mm}^{3}$ volume shown in red so that the DNS results are not biased by the $\mathrm{N}_{2}$ co-flow. The figure is adopted from [30].

field generated prior to the simulation, based on a prescribed Passot-Pouquet energy spectrum [31]. As empirical input, the stagnation point location used in Eq. 12 was taken to be the mean DNS stagnation location, $4.8 \mathrm{~mm}$. Additionally, ODT has three adjustable parameters that need to be specified:

- Viscous penalty parameter $Z=0.1$.

- Eddy frequency parameter $C=3.5$.

- Maximum eddy size allowed is $5 \mathrm{~mm}$, which corresponds to $1.3 \cdot L_{11}$.

These parameters were chosen by matching spatial and state-space statics to

DNS results. A parameter sensitivity study is conducted in section 4.3

\subsection{DNS physical and numerical parameters}

The DNS set-up is detailed in [30], here only the key points are re-stated. The three-dimensional physical extent of the computational domain is $0.95 D \times$ $1.48 D \times 1.48 D$ and the domain is discretized into $432 \times 640 \times 640$ grid cells in the 

three directions where the resolution adequately resolves both the flame and turbulent flow field, resulting in a uniform spacing of $D / d x=470$. To produce the reactant side turbulent inlet conditions, velocity fluctuations are superimposed on the mean inlet velocity at the reactants stream inlet. These fluctuations are three-dimensional DNS of a non-reactive homogeneous isotropic field performed with the Sandia 3D Direct Numerical Solver S3D [32]. A homogeneous isotropic turbulence field is initially generated using the method described by [33], based on a prescribed Passot-Pouquet energy spectrum [31] that satisfies continuity and subsequently evolves until turbulence is established.

\section{Results}

In this section a macroscopic description of the overall flame burning behavior is provided from a statistical description of the turbulent flame and results from ODT and DNS are compared. The Favre mean of a variable, $\widetilde{\phi}$, is defined as $\widetilde{\phi}=\overline{\rho \phi} / \bar{\rho}$ where the overbar denotes ensemble temporal averaging defined as:

$$
\phi(x, y)=\frac{1}{N_{t}} \sum_{n=1}^{N_{t}} \phi\left(x, y, t_{n}\right) .
$$

$N_{t}$ is the number of samples in the statistically stationary period in the simulation over which ensemble averaging is performed.

The results section is outlined as follows: in sec. 4.1 the 1D laminar strained flame results are presented. First, the evolution of the maximum temperature and maximum heat release rate are presented as a function of bulk strain rate. Then, for the bulk strain rate of the current counterflow configuration the 1D laminar strained flame results as a function of the nozzle separation distance are shown. In sec. 4.2.1 spatial statistics of the turbulent flame are compared between ODT and DNS results. In sec. 4.2.2 mixing rates are compared by looking at the scalar dissipation rate. In sec. 4.2.3, flame extinction/ignition characteristics are discussed. Sec. 4.2.4 compares scatter plots of heat release 
rate and species conditioned on temperature to show the range of results obtained by ODT and DNS. Sec. 4.2.5 tests for superadiabaticity by showing the equilibrium temperature reached for mixture states taken from the DNS and ODT results. Sec. 4.2.6 looks at the effects of differential diffusion. For a more stringent comparison between ODT and DNS, sec. 4.2.7 compares the mean and RMS profiles of the scatter plots. Finally, in sec. 4.3 a sensitivity study to ODT input parameters is presented.

\subsection{Laminar Flame Results}

Prior to the application of ODT to the turbulent counterflow flame, the response of the flame to strain rate fluctuations was examined using laminar flame simulations. The laminar ODT simulations include the advection and the dilatation model, but not the ODT eddy events nor the Darrieus-Landau instability model. For these ODT simulations, the strain rate was progressively increased from $200 \mathrm{~s}^{-1}$ to $10,000 \mathrm{~s}^{-1}$, while the composition and temperature of the counterflowing streams and the nozzle separation distance were held constant and identical to the three-dimensional turbulent flame parameters investigated with DNS. The dependence of the maximum heat release rate and maximum temperature on bulk strain rate is shown in Fig. 4. Figure 4 shows that there is a non-monotonic dependence of heat release rate and temperature on strain rate. For low-to-moderate strain rate, up to approximately $2,400 \mathrm{~s}^{-1}$, the peak heat release rate increases with increasing strain rate as expected from effects of nonequidiffusivity [34]. At higher strain rate, the flame is pushed closer to the stagnation plane and the temperature and heat release rate decrease with increasing strain rate. The maximum temperature does not decrease below $1,475 \mathrm{~K}$, as this is the product side inlet temperature. The flame response to strain rate as shown in Fig. 4 is described by a stretched S-curve, as opposed to a folded S-curve when extinction is abrupt [35, 36]. The gradual extinction occurs due to the temperature of the product stream, which is higher than the adiabatic temperature of the lean premixed flame and thus provides back support that prevents the flame from extinguishing abruptly. The stretched S-curve 


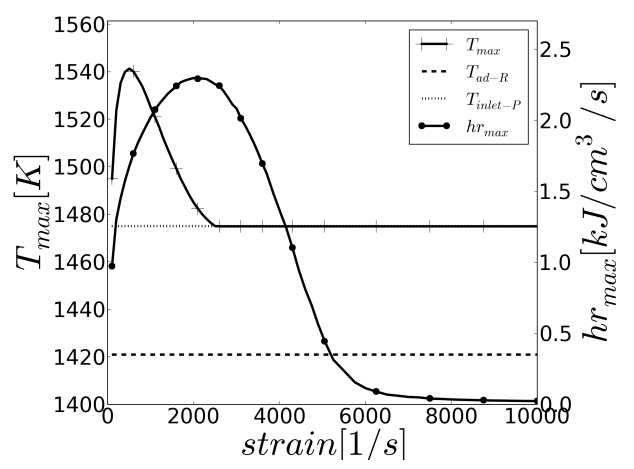

Figure 4: Steady state solutions of the 1D laminar strained flame as a function of bulk strain rate. ODT results for maximum heat release rate and maximum temperature are plotted. Additionally, the reactant side adiabatic flame temperature, $T_{a d-R}$, and the product side inlet temperature, $T_{\text {inlet-P }}$, are shown.

lacks a turning point and results in an ambiguous definition of the extinction limit and the corresponding extinction strain rate. Therefore, in the present study, following [30], the flame is considered to be extinguished when the instantaneous heat release rate is lower than 0.5 percent of the maximum heat release rate of the strained laminar case which corresponds to $0.01 \mathrm{~kJ} / \mathrm{cm}^{3} / \mathrm{s}$.

In Fig. 5. ODT results for the one-dimensional strained laminar flame $\left(\mathrm{a}=2,400 \mathrm{~s}^{-1}\right)$ are compared to results from the OPPDIF solver of the CHEMKIN package [37]. This strain rate corresponds to the bulk strain rate of the current counterflow configuration. Results are centered about the stagnation point, with OPPDIF results offset by $0.078 D$ to obtain a clearer comparison. It can be seen that the normalized axial velocity profile $u / U_{u}$ decreases from unity at the reactants nozzle to -3.45 at the counterflowing product stream nozzle tip. Within the domain, the ODT velocity profile, and therefore local strain rate, underestimates the OPPDIF results. This shows that our linear approximation for the pressure source term in Eq. 12 does not accurately reflect the spatial variation of pressure in OPPDIF. However, near the stagnation point $x / D=0$ the velocity profile shows good agreement with OPPDIF data. In this region the dilatation model has a large influence on the velocity field. The temperature 

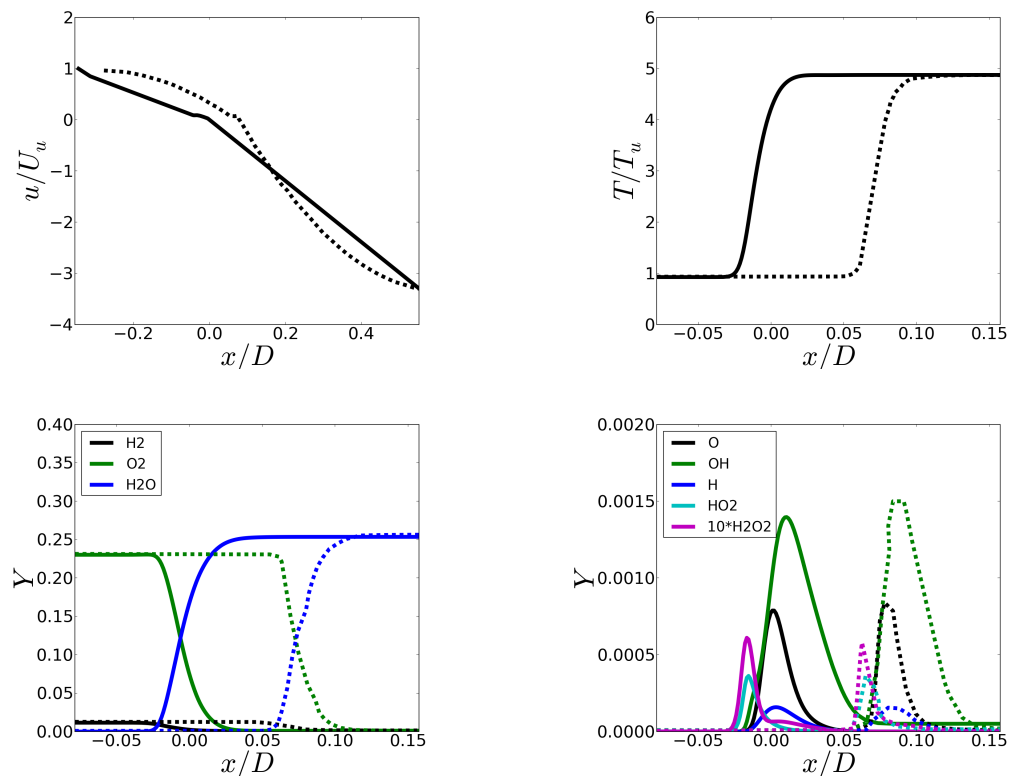

Figure 5: Laminar strained flame results. Top: Axial velocity (left) and temperature (right) as a function of the axial distance normalized by the jet diameter. The velocity $u$ is normalized by the bulk velocity of the reactants $U_{u}$ and the temperature by the reactant inflow temperature $T_{u}$. Bottom: Major (left) and minor (right) species mass fractions as a function of the axial distance. Reactant and product streams are on the left and right side of the plot, respectively. On the x-axis, zero is the stagnation point location. The solid lines represent the ODT results and the dashed lines the corresponding OPPDIF results. To obtain a clear visual comparison, OPPDIF results are offset by $0.078 D$ in all plots and temperature and species plots are zoomed in.

and major and minor species profiles are almost identical. Due to the previously mentioned lower strain rate encountered by ODT, a very slight discrepancy is observed, whereby the ODT profiles are more rounded. Here, the lower strain rate allows diffusion to broaden the ODT curves slightly more.

\subsection{Turbulent Flame Results}

\subsubsection{Spatial Comparisons}

Favre velocity and scalar means and variances are presented in this section. The Favre mean and RMS of the normalized axial velocity, temperature, and 
major $\left(\mathrm{H}_{2} \mathrm{O}, \mathrm{H}_{2}, \mathrm{O}_{2}\right)$ and minor $\left(\mathrm{O}, \mathrm{OH}, \mathrm{H}, \mathrm{H}_{2} \mathrm{O}_{2}, \mathrm{HO}_{2}\right)$ species as a function of the stagnation point location are presented in Fig. 6] It can be seen that the mean ODT and DNS velocity profiles are very similar to the laminar strained flame ODT and OPPDIF velocity profiles respectively. The ODT and DNS normalized RMS of the axial turbulent fluctuations is $\widetilde{u_{r m s}} / U_{u}=35 \%$ at the exit of the reactant jet.

In the DNS, the normalized RMS of the axial turbulent fluctuations increases by a factor 1.85 towards the stagnation plane, attaining a maximum value at $x / D=0.06$, and vanishing in the product stream as expected. In ODT, the RMS first decreases and then recovers to approximately the same level at the stagnation plane. For ODT, the fluctuations decrease from the inlet because we apply a constant pressure source term that decreases the velocity amplitude from the inlet towards the stagnation point. Near the stagnation point, the generated turbulence then increases the fluctuations.

The Favre mean normalized temperature $\widetilde{T} / T_{u}$ increases monotonically between unity and five between the cold and hot boundaries and is approximately 2.5 at the stagnation plane. At this axial location, the normalized RMS approaches $97 \%$ of its maximum value of 1.72 for the DNS while for ODT it approaches its maximum value of 1.57. The distribution of the mean temperature and its fluctuations reveal that the mean thickness of the mixing layer between the cold reactant and the hot product stream is $0.41 D$ for the DNS, while it is $0.69 D$ for ODT. The mixing layer is defined to start at the location where $\widetilde{T}_{R M S}$ reaches $1 \%$ of $T_{u}$ and ends where $\widetilde{T}_{R M S}$ drops again below this value. For ODT, the broadness of the thickness is directly related to the maximum allowed eddy size of $0.39 D(5 \mathrm{~mm})$, as the extent of the temperature fluctuation manifests itself $0.39 D$ from the stagnation point into the product side. Therefore, if the maximum eddy size was set larger, intermittency would result in eddies that broaden the mixing layer even more (see Sec. 4.3). The significant levels of temperature fluctuations, i.e. up to $500 \mathrm{~K}$ for DNS and $460 \mathrm{~K}$ for ODT, correlate with the spatially and temporally intermittent flame attenuation events that occur near the stagnation plane. The heat release rate 
and radical production rates are sensitive to temperature fluctuations, amplified through the Arrhenius chemical effect.

Major species mean profiles vary monotonically between the two nozzles while minor species feature distributions centered around the stagnation plane. Contrary to the 1D laminar flame profiles at the same bulk strain rate, the mean minor-species spatial distributions exhibit maxima that are nearly collocated and the extent of the reaction zone is approximately 2.3 times wider than the corresponding laminar flame. This is expected as Karlovitz and the Damköhler number are $K a=26$ and $D a=1.2$ respectively, which indicate that the current turbulent flame is in the thin reaction zone regime. The smallest eddies can enter the reactive-diffusive flame structure, however these small eddies are still 440 larger than the reaction zone thickness and can therefore not penetrate into that layer. The second moments of all species are zero at the jet exits and increase with axial distance, attaining their maximum in the vicinity of the stagnation plane where the flame resides.

Comparing ODT temperature and species profiles with DNS, we see that although ODT is a reduced order model, it is able to achieve a good quantitative comparison for both mean and RMS temperature and species results. RMS profiles for temperature and major species are slightly underpredicted. This can be explained by noting that the axial movement of the stagnation point is larger for the DNS than for ODT. In the DNS the mean stagnation point is located $4.8 \mathrm{~mm}$ from the reactant side nozzle, and this was used as empirical input for ODT (see Sec. 3.1). The RMS of the stagnation point location is $0.06 D$ and $0.04 D$ in the DNS and ODT respectively. For the DNS, the larger stagnation plane motion increases RMS values but is not mixing. In ODT, the axial movement of the stagnation plane is seen to a lesser degree due to the prescribed constant pressure shape function for the advection velocity. Minor species mean profiles however, have peaks about two times that of the corresponding DNS peaks. This indicates that burning in ODT is more localized about the stagnation plane. 

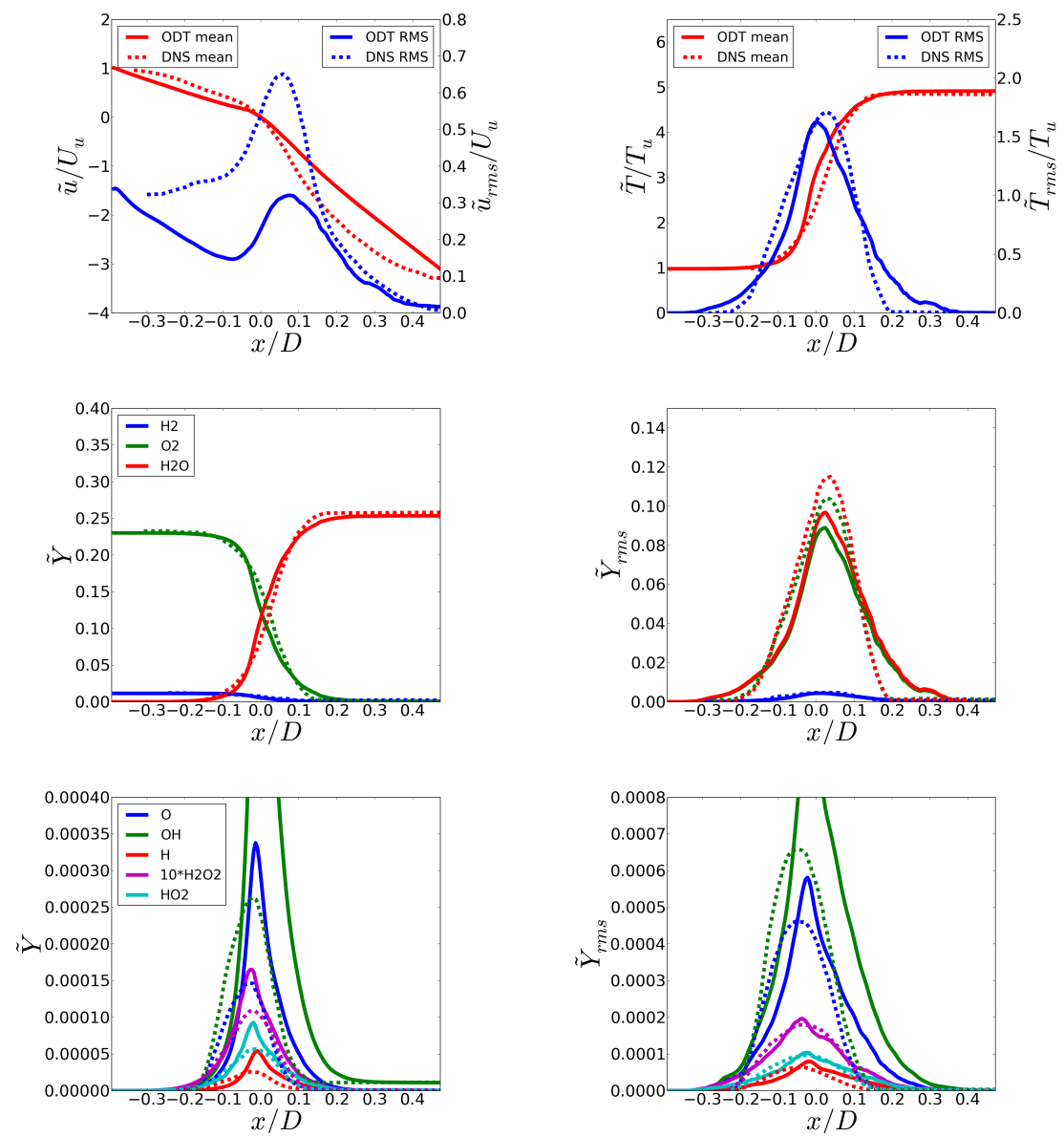

Figure 6: Top: Favre mean and RMS of the axial velocity and temperature as a function of the axial distance normalized by the jet diameter. The velocities are normalized by the bulk velocity of the reactants $U_{u}$ and the temperature by the reactant inflow temperature $T_{u}$. Density weighted major (middle) and minor (bottom) mean (left column) and RMS (right column) species mass fractions as a function of the axial distance normalized by the jet diameter. Solid and dotted lines represent ODT and DNS results respectively. Results are offset on the $x$ axis, such that $x / D=0$ is the stagnation point for both ODT and DNS. 

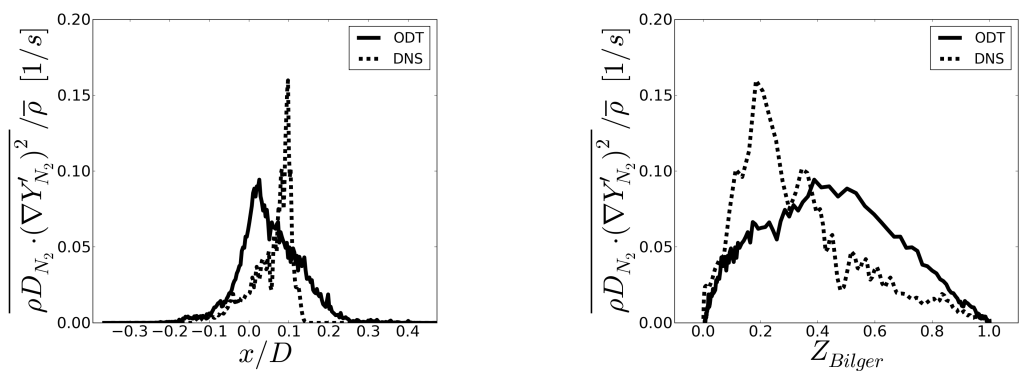

Figure 7: Favre averaged scalar dissipation rate as a function of the axial distance normalized by the jet diameter (left) and as a function of Bilger's mixture fraction (right). Solid and dotted lines represent ODT and DNS results respectively. Spatial results are offset on the $x$ axis, such that $x / D=0$ is the stagnation point for both ODT and DNS.

\subsubsection{Scalar Dissipation Rate Comparisons}

The scalar dissipation rate can be physically interpreted as a mixing rate, or equivalently as a rate at which scalar fluctuations are destroyed [38]. Therefore, to look at mixing decoupled from burning we next present Favre averaged results of the nitrogen dissipation rate. The nitrogen concentration differs in the reactant and product inlet streams and therefore provides a simple conserved scalar that is representative of conserved scalar dissipation rates. In Fig. 7 we plot the Favre averaged scalar dissipation rate as a function of axial distance and as a function of Bilger's mixture fraction [39]. Spatial results show that ODT is in reasonable agreement with the DNS although it underestimates peak mixing levels. The degree of accuracy of ODT seen in Fig. 7 is consistent with, and an important determining factor of, the accuracy of the statistics of various thermochemical quantities that are presented here. Additionaly, this demonstrates that the ODT formulation captures relevant fluctuation properties of full 3D turbulence in 1D.

\subsubsection{Flame Attenuation}

The nature of extinction we observe in this flame is not abrupt extinction of flame regions, but rather gradual attenuation. A flame experiences attenuation 
when portions that are supposed to be burning vigorously experience a drop in heat release rate values. To provide a measure of this, we first define a progress variable: $p . v .=\left(Y_{H_{2}}-Y_{H_{2}, u}\right) /\left(Y_{H_{2}, u}-Y_{H_{2}, b}\right)$, where $Y_{H_{2}, u}$ and $Y_{H_{2}, b}$ are the reactant and product side inlet $\mathrm{H}_{2}$ mass fractions respectively. In Fig. 8 (left) we plot heat release rate against p.v. for the 1D strained laminar flame computed with OPPDIF. From this curve we see that peak heat release rate for the laminar flame is reached in the vicinity of $p . v$. in range from 0.5 to 0.6 . This p.v. range demarcates portions that should be burning well. In Fig. 8 (right) we plot the probability density function (pdf) of heat release rate on the center line conditioned on being in this p.v. range. The heat release rate range for the laminar strained flame (dotted line) in this p.v. range is depicted from the left plot for comparison. The DNS result (dashed line) shows lower heat release rate values which denote significant attenuation. The ODT pdf (solid line) shows excellent agreement with the DNS data. This gives a quantitative demonstration that attenuation characteristics in ODT are captured well. The DNS deviations from the ODT curve are mainly sharp peaks and troughs, possibly reflecting the greater statistical variability of the DNS relative to ODT rather than ODT modeling error. We also checked the heat release rate pdf curve conditioned on additional p.v. ranges as well (not shown): 0.3-0.4, 0.4-0.5 and 0.6-0.7. These indicated some quantitative variations of heat release rate pdf shape from range to range, but at low heat release rate they all indicate the same rough powerlaw dependence seen for p.v. in the range 0.5-0.6, and also they all show good agreement between ODT and DNS.

\subsubsection{Scatter Plot Comparisons}

Temperature-conditioned statistics are widely used to analyze the statespace structure of turbulent flames because they conveniently illustrate effects of finite-rate kinetics that cause the thermochemical state to deviate from equilibrium. For ODT specifically, such statistics obtained from non-premixed configurations have proven useful and instructive [13, 14]. In addition to the novel features of the present ODT formulation (see Sec. 2.3), this study serves as the 

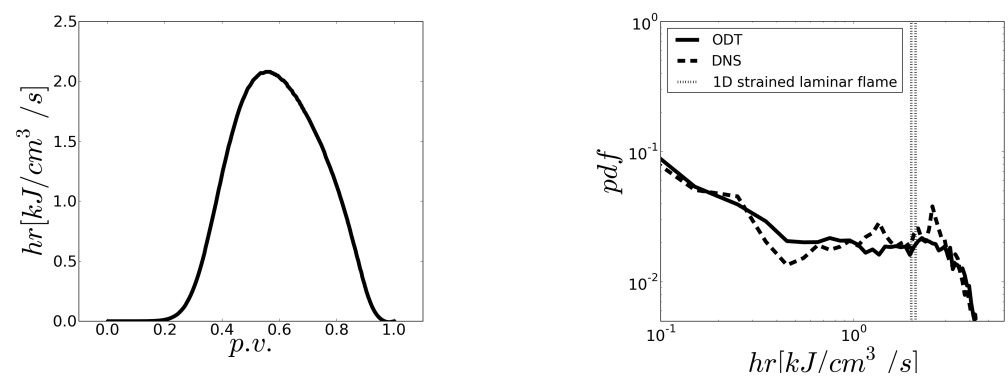

Figure 8: Left: Heat release rate on the center line plotted against progress variable (p.v.) for the 1D strained laminar flame computed with OPPDIF. The progress variable is defined as p.v. $=\left(Y_{H_{2}}-Y_{H_{2}, u}\right) /\left(Y_{H_{2}, u}-Y_{H_{2}, b}\right)$, where $Y_{H_{2}, u}$ and $Y_{H_{2}, b}$ are the reactant and product side inlet $\mathrm{H}_{2}$ mass fractions respectively. Right: Probability density function (pdf) of heat release on the center line conditioned on the p.v. in range 0.5-0.6. Solid line (ODT), dashed line (DNS), and the 2 dotted lines represent the heat release rate range for the 1D strained laminar flame in range p.v. between 0.5 and 0.6 (taken from the left plot).

first detailed presentation of such statistics from ODT simulations of premixed combustion.

In Fig. 9and Fig. 10 we compare ODT and DNS scatter plots of heat release rate and major and minor species mass fractions conditioned over temperature. Each value plotted represents a specific point in time and space. For the DNS, values from the center line were taken over 10 residence times 100 times per residence time, while for ODT, values over the entire domain were taken over 100 residence times 10 times per residence time. Therefore, the number of times the data was sampled at is the same for DNS and ODT, however ODT sampled for a longer run time but less frequently. The values for the corresponding strained $\left(a=2,400 s^{-1}\right)$ laminar flame are shown for reference. The plots demonstrate that ODT is able to capture the full range of results seen by the DNS over the entire temperature range. It is observed that the calculation points for ODT are distributed in a band width that is in good agreement with DNS data at all temperatures. The most noticeable difference between ODT and DNS is in the heat release rate plot at temperatures below 1,000 K. Here, a small number of points show higher heat release rates than indicated by DNS. A possible 
explanation is that this higher heat release rate level at low temperatures is an artifact of the instantaneous mappings, whereby unrealistic gradients are momentarily created that could affect the diffusion process. This shows that due to the stochastic nature of ODT, certain states can be obtained that are otherwise not obtained when the full Navier-Stokes equations are solved.

Scatter plots for major species $\mathrm{H}_{2} \mathrm{O}$ and $\mathrm{O}_{2}$ compare well between DNS and ODT. The comparison for $\mathrm{H}_{2}$ shows that: 1) at temperatures below 1,000 K, DNS has more points below the laminar line and 2) in general ODT has a wider band above the laminar line at all temperatures.

In general, in the DNS we see distinct structures at high temperatures that are not necessarily so prominent in the ODT results. However, this is not a case of ODT not capturing these states, but rather that ODT captures additional states to those seen by the DNS that blur out the distinct shapes. Specifically looking at Fig. 9, and comparing the $\mathrm{O}_{2}$ plots, we see a distinct structure in the DNS at temperatures between 1,500 - 1,700 K. In ODT, results in this temperature range are more broadly and smoothly spread than in the DNS. A possible explanation for this is that ODT simulated more diverse residence times than the DNS and therefore sees more states which blur out the sharp features in the DNS.

\subsubsection{Superadiabaticity Test}

In Fig. 9] states with temperature up to approximately 1,700 K are obtained.

${ }_{545}$ This is considerably higher than the product side inlet temperature of $1,475 \mathrm{~K}$ and also above the reactant side adiabatic flame temperature of $1,420 \mathrm{~K}$. To test for superadiabaticity, temperature that exceeds the highest equilibrium temperature that is possible for any mixture of the two inlet states, we take each state above $500 \mathrm{~K}$ presented in Fig. 9] as an input condition (pressure, temperature and species) and perform an equilibrium calculation. The equilibrium temperature reached versus input temperature from DNS and ODT is shown in Fig. 11 Points on the diagonal line indicate no change in temperature (i.e. input mixture is at equilibrium). Points above the diagonal line indicate a temperature 

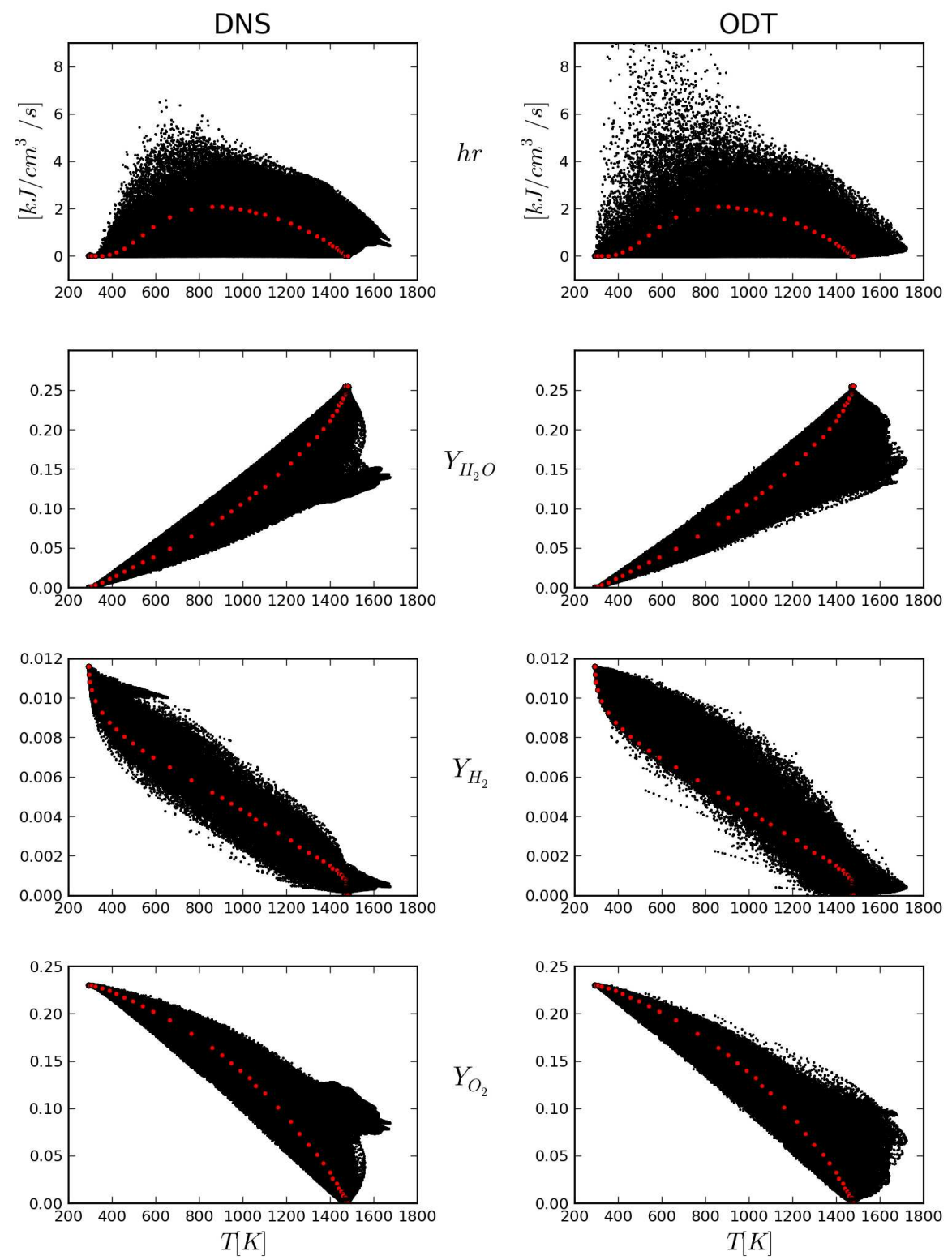

Figure 9: Scatter plots of heat release rate and major species mass fractions with temperature on the burner centerline. DNS (left), ODT (right). The red symbols represent the corresponding 1D strained laminar flame computed with OPPDIF. 

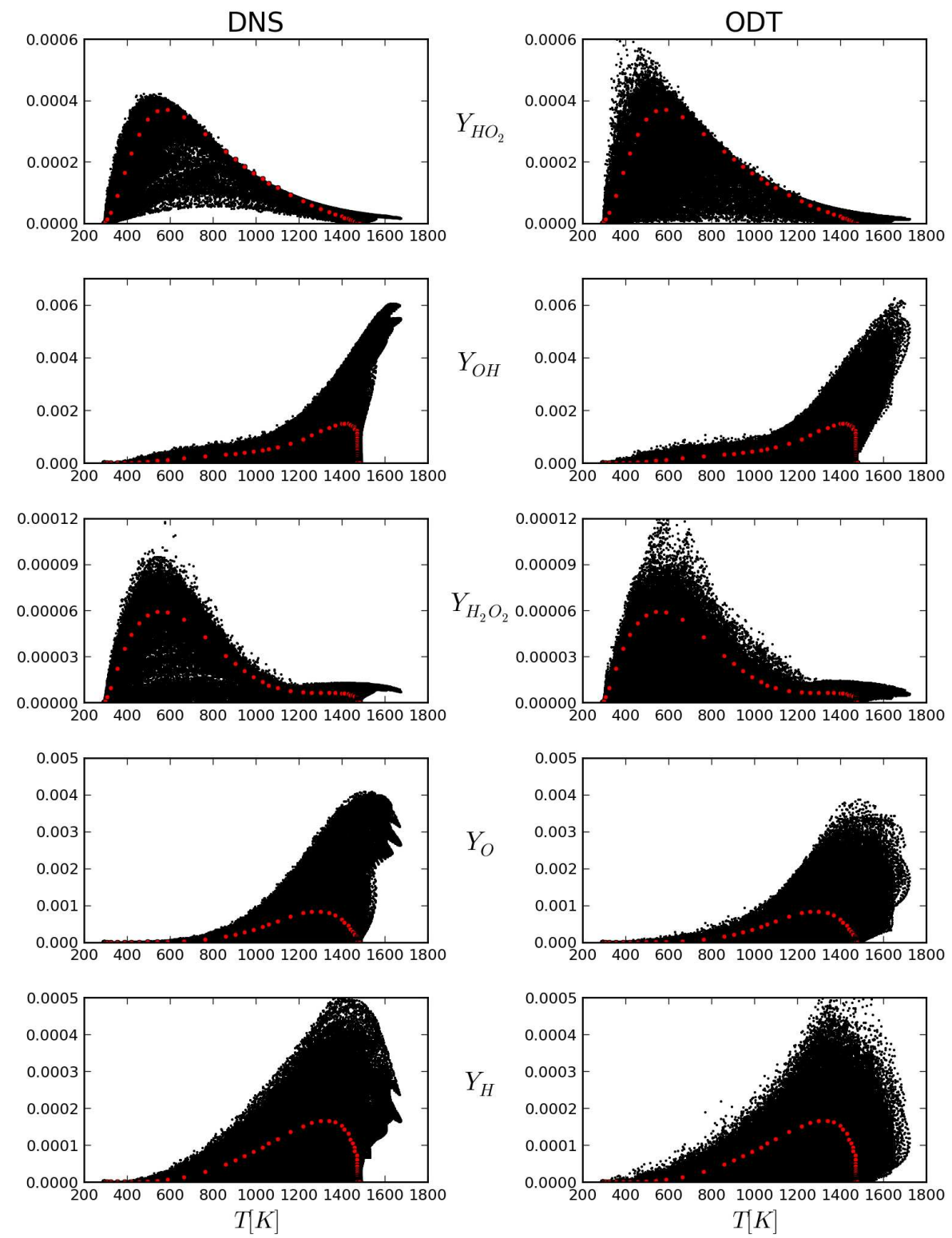

Figure 10: Scatter plots of minor species mass fractions with temperature on the burner centerline. DNS (left), ODT (right). The red symbols represent the corresponding 1D strained laminar flame computed with OPPDIF. 

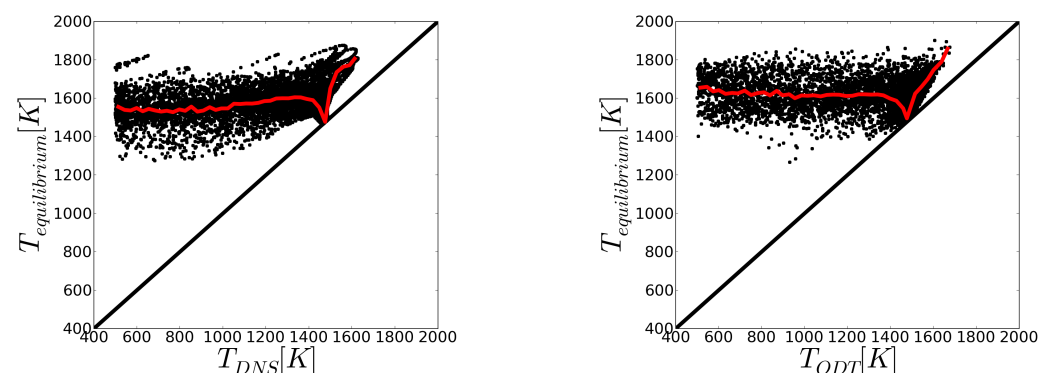

Figure 11: Equilibrium calculation for input states from DNS (left) and ODT (right) results. The red line represents the average equilibrium temperature for given input temperature.

rise at equilibrium and points below indicate a temperature drop. No points are seen below the diagonal line for both DNS and ODT, indicating that superadiabaticity is not reached. The equilibrium temperature remains the same as the input temperature only for a group of points with initial temperature around $1,475 \mathrm{~K}$. These represent the product side inlet gas that is input at a state of equilibrium. All other points represent a mixture of the reactant and product side inlet states.

To facilitate the comparison between ODT and DNS results, the average equilibrium temperature reached for input temperature is additionally shown in red in Fig. 11, Comparing the ODT and DNS average equilibrium temperatures shows that for input temperatures below 1,000 K, ODT reaches higher equilibrium temperatures. Differences in ODT and DNS species results below $1,000 \mathrm{~K}$ have been highlighted in Sec. 4.2.4 here it is only noted that differences between ODT and DNS input states are amplified through the equilibrium calculation and lead to larger differences in the equilibrium states reached. Above $1,000 \mathrm{~K}$, the average equilibrium temperatures are similar.

\subsubsection{Differential Diffusion Effects}

Fig. 9 and 10 show that the turbulent flame reaches temperatures up to approximately $1,700 \mathrm{~K}$, whereas the laminar flame reaches a maximum peak temperature of $1,540 \mathrm{~K}$. A possible explanation for the turbulent flame reaching higher temperatures is that these temperatures are associated with differential 

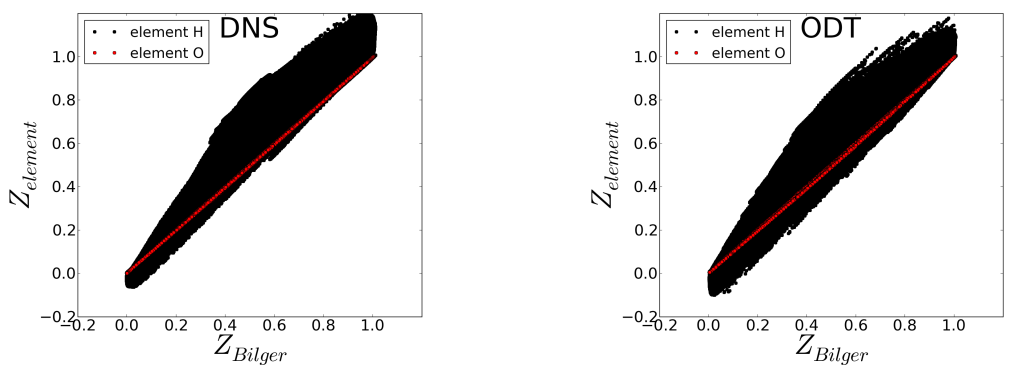

Figure 12: Scatter plot of element O (red) and element H (black) mixture fraction vs. Bilger's mixture fraction for DNS (left) and ODT (right).

diffusion (DD). Low molecular weight hydrogen $(1.0 \mathrm{~kg} / \mathrm{kmol})$ species diffuse more rapidly than heavy oxygen $(16.0 \mathrm{~kg} / \mathrm{kmol})$ and nitrogen $(14.01 \mathrm{~kg} / \mathrm{kmol})$ species, in some instances increasing the $\mathrm{H}_{2} / \mathrm{O}_{2}$ ratio creating a richer mixture. To validate this claim, in Fig. 12 we first compare DNS and ODT results for element mixture fraction $\mathrm{H}$ and $\mathrm{O}$ versus Bilger's mixture fraction. We see that for both the DNS and ODT element $\mathrm{H}$ mixture fraction has values greater than and less than 1, while the Bilger's mixture fraction has values strictly between 0 and 1. This, and noting the difference between element $\mathrm{H}$ and element $\mathrm{O}$ mixture fraction, shows that DD effects are present. ODT results compare well to DNS data.

Having validated the ODT DD results, ODT is now re-run with the Lewis number of all species set to 1. Fig. 13 shows the scatter plot of heat release rate with temperature on the burner centerline and we see that temperatures above the laminar flame temperature are not obtained. This is an illustration that having validated ODT, we can do parameter variations to look at questions of interest more quickly and easily than by running additional DNS cases.

\subsubsection{State-Space Comparisons}

For a more stringent comparison between ODT and DNS, mean and RMS plots conditioned on temperature for heat release rate and major and minor species are presented. A qualitative agreement is achieved in all of the cases, 


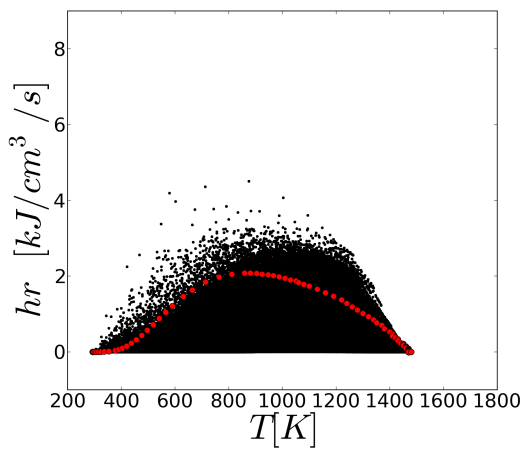

Figure 13: ODT scatter plot of heat release rate with temperature on the burner centerline with Lewis number of all species equal to 1 . The red symbols represent the corresponding 1D strained laminar flame computed with OPPDIF.

with quantitative agreement for some of the mean and RMS profiles. In Fig. 14 heat release rate conditioned on temperature is compared. For reference, the strained laminar flame results are also shown. ODT mean results compare well to DNS for temperatures above 1,000 K. For temperatures below 1,000 K, ODT underestimates the heat release rate. A possible explanation for this is that in low temperature regions, the flame is weaker and more sensitive to strain fluctuations. ODT through its instantaneous maps can induce momentarily artificially high strains which disrupt the preheating process. RMS profiles show good qualitative comparison to DNS throughout the temperature range.

In Fig. 15, species conditioned on temperature are compared. In all cases, ODT gives good qualitative results for both mean and RMS profiles. From the plots we see that at low temperatures, below $1,000 \mathrm{~K}$, reactions involving the formation and consumption of $\mathrm{HO}_{2}$ and $\mathrm{H}_{2} \mathrm{O}_{2}$ chemistry are important. Mean profiles here are again underestimated showing underignition at low temperatures.

\subsection{Parameter Sensitivity Study}

The DNS data is used to provide guidance on the selection of the ODT parameters listed in section 3.1. Sensitivity analysis is performed in the con- 


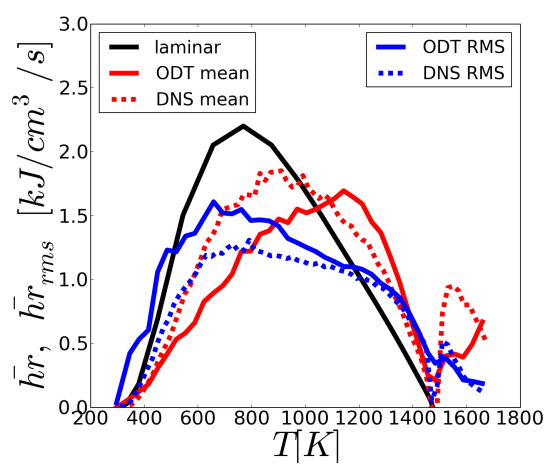

Figure 14: Temperature conditioned mean (red) and RMS (blue) of the heat release rate. Solid lines: ODT, dotted lines: DNS. The black line represents the corresponding 1D strained laminar flame computed with OPPDIF.

text of Favre averaged spatial statistics and by collecting state-space statistics conditioned on temperature over 10 residence times from the DNS and ODT simulations. Statistical convergence has been ensured by verifying that the statistics from half the samples are indistinguishable from those from the full samples. The sensitivity of the ODT results to the eddy frequency parameter $C$, the maximum allowed eddy size $L_{\max }$, and the stagnation point location is presented in Fig. 16. Favre averaged temperature fluctuations and heat release rate conditioned on temperature are shown because they characteristically represent spatial and state-space statistics. A sensitivity study for the viscous penalty parameter $Z$ is not shown, as $Z$ was chosen small enough such that results are insensitive to it.

The upper row in Fig. 16 shows results for $C=1,3.5$, and 10, the middle row shows results for $L_{\max }=3,5$, and $7 \mathrm{~mm}\left(L_{\max } / D=0.24,0.39\right.$, and 0.55$)$, and the bottom row shows results for setting the stagnation point location to $+/-1 \mathrm{~mm}$ of the nominal case. These parameters are chosen to cover a relatively broad range of values and to show the sensitivity of results to changes in these values. We first note, that for all values tested, results remain qualitatively similar indicating that simulation results are insensitive to moderate changes in parameter values. The temperature RMS plot shows that as $C$ increases, the 

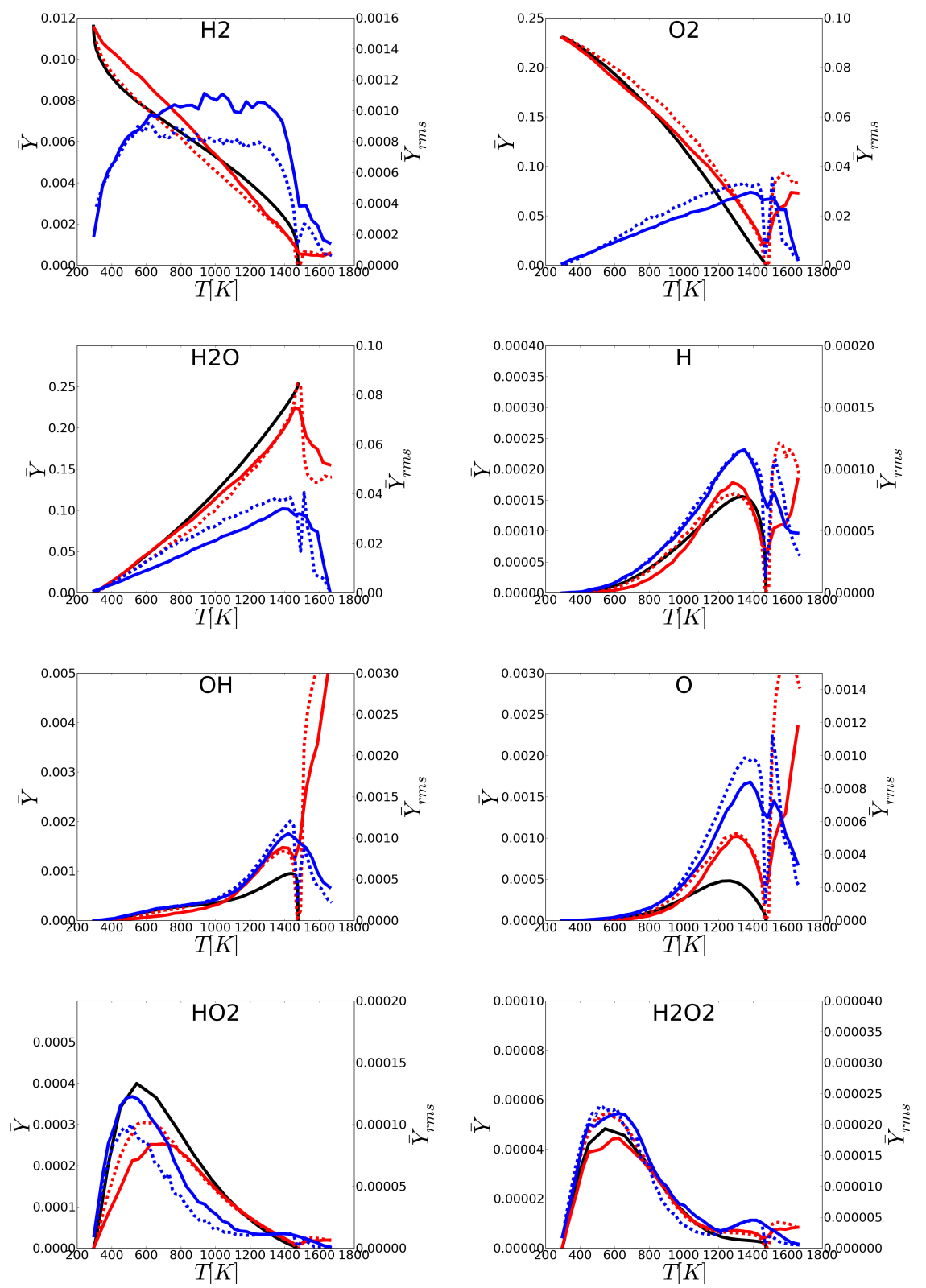

Figure 15: Temperature conditioned mean (red) and RMS (blue) of mass fractions for all major and minor species are shown. Solid lines: ODT, dotted lines: DNS. The black line represents the ODT results of the strained laminar flame with a strain rate of $2,400 \mathrm{~s}^{-1}$. 
mixing layer broadens. A broadening of the mixing layer is similarly obtained by increasing the maximum size of eddies allowed from 3 to $7 \mathrm{~mm}$. Although $L_{\max }$ increases by only a factor of 2.3 from 3 to $7 \mathrm{~mm}$, it has a larger influence on the mixing width than increasing $C$ from 1 to 10 . The heat release rate statistics however show the reverse effect, whereby results vary only slightly with changes in $L_{\max }$, but more prominently with changes in $C$. As $C$ is increased, particularly at temperatures below $1,200 \mathrm{~K}$, the heat release rate decreases indicating higher levels of extinction. This is expected, as increasing $C$ is representative of increasing the turbulence intensity at all length scales. If the turbulence intensity becomes too high, then within the residence time of the current counterflow configuration, a flame can not be sustained. Although, changes in $L_{\max }$ affect heat release rate results only slightly, we do see that as $L_{\max }$ increases, the heat release rate rises. Larger eddies simultaneously mix in larger quantities of fresh gas and also increase the residence time of the gas by displacing burning fronts from the stagnation point towards the nozzles, giving the mixture more time to ignite. For the ODT counterflow simulations, the stagnation point location is an empirical input which is taken from the DNS. The sensitivity study shows that spatial and state-space statistics are not greatly affected by moderate changes in the stagnation point location.

\section{Conclusions}

In summary, the ODT methodology was applied to a turbulent reactant-toproduct counterflow flame. Configuration specific models needed to address the 3D dilatation and advection phenomena on the 1D line were presented.

Comparison of results for the laminar strained flame obtained from ODT and OPPDIF, shows that the strain rate produced by ODT is slightly lower than that produced by OPPDIF. As a consequence, a very slight discrepancy is observed between ODT and OPPDIF profiles, whereby ODT profiles are more diffused.

Comparing ODT results with DNS data for spatial mean and fluctuating 

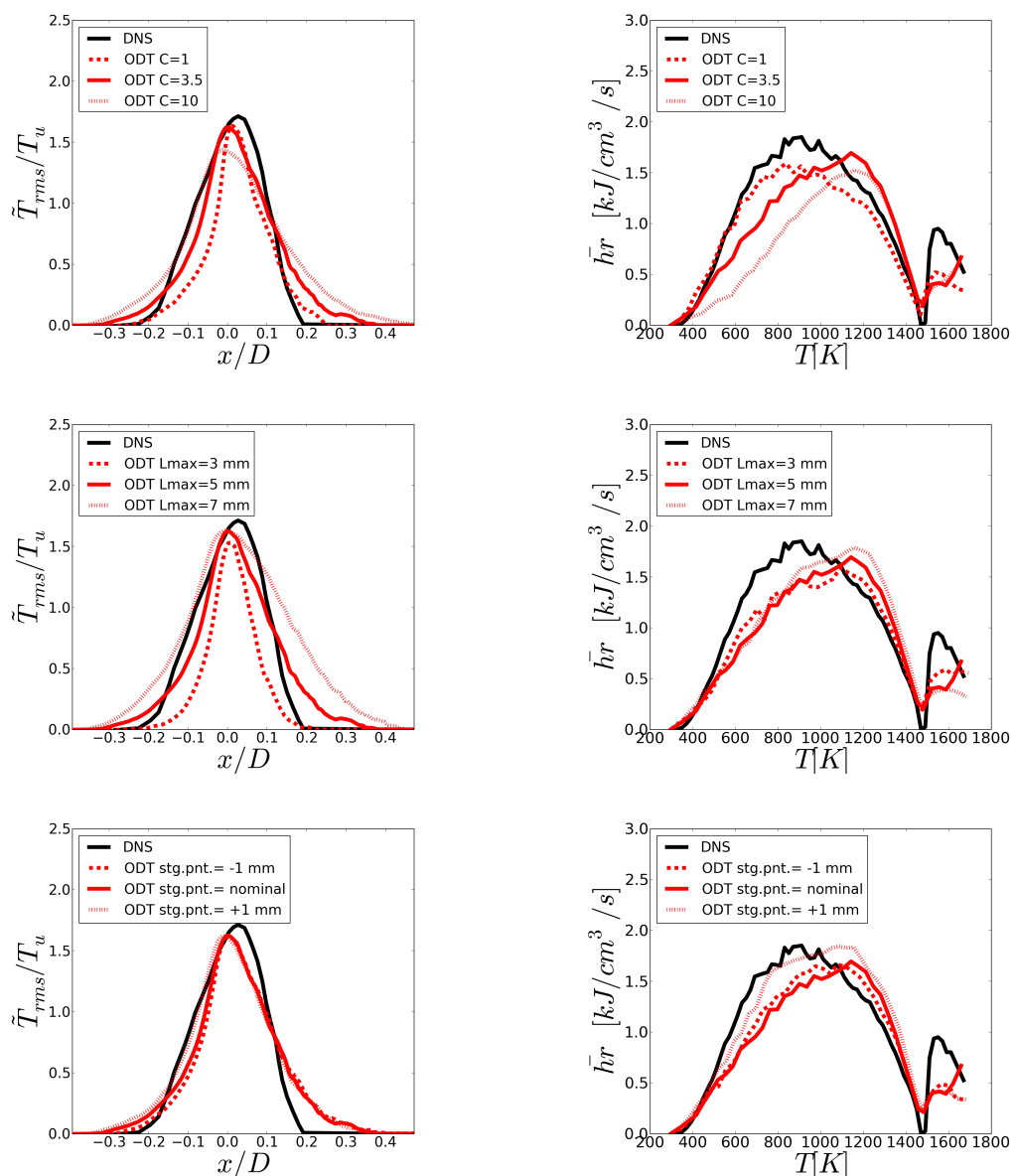

Figure 16: Sensitivity of the ODT results to the eddy frequency parameter $C$ (top), maximum allowed eddy size $L_{\max }$ (middle), and stagnation point location (bottom). The DNS data are also plotted for reference. Left: Favre RMS of temperature, normalized by the reactant inflow temperature $T_{u}$ as a function of the axial distance normalized by the jet diameter. Right: mean heat release rate conditioned on temperature. 
velocity, temperature, and major and minor species profiles, show that ODT results for temperature and species mean and RMS profiles are in good agreement with DNS. Comparison of the scalar dissipation rate shows that ODT estimates the mixing level seen in the DNS reasonably well. Flame extinction and ignition characteristics were compared by looking at the probability distribution of heat release rate conditioned on a progress variable. Comparisons show that the flame attenuation characteristics are captured accurately in ODT.

Scatter plots for heat release rate and major and minor species as a function of temperature were presented to illustrate model capabilities in capturing the full range of results observed by the DNS. Scatter plot results showed that temperatures above the hot product side inlet temperature and above the adiabatic flame temperature of the reactants are obtained. A test for superadiabaticity showed that superadiabatic conditions are however not reached. An additional study showed that differential diffusion effects are responsible for the higher temperatures reached in the turbulent flame.

State-space statistics of heat release rate and all species conditioned on temperature were shown for a more stringent comparison of ODT and DNS data. Good agreement with the DNS results is attained, although heat release rates for temperatures below $1,000 \mathrm{~K}$ are underpredicted.

680 A sensitivity study to ODT input parameters was carried out by varying the eddy frequency parameter $C$, the maximum eddy size allowed $L_{\max }$, and the stagnation point location. For the range of parameters tested, results remained qualitatively consistent with moderate quantitative changes.

This work has demonstrated the application of ODT to the counterflow configuration. Results show that although ODT is a reduced order model, qualitative and quantitative agreement with to DNS data are obtained.

\section{Acknowledgements}

The work at Sandia National Laboratories was sponsored by the US Department of Energy, Office of Basic Energy Sciences, Division of Chemical Sciences, 
Geosciences, and Biosciences. Sandia National Laboratories is a multiprogram laboratory operated by Sandia Corporation, a Lockheed Martin Company, for the US Department of Energy under contract DE-AC04-94-AL85000. This research used resources of the National Energy Research Scientific Computing Center, which is supported by the Office of Science of the U.S. Department of Energy under Contract No. DE-AC02-05CH11231.

\section{Appendix: Reference-frame acceleration in ODT}

The Darrieus-Landau (DL) instability model described in Sec. 2.3.3 is the first demonstration of a strategy for incorporating flow acceleration effects into ODT. Another such effect that might be incorporated in the future is shockturbulence interaction. To provide context for the present DL formulation and related future model extensions, the physical and formal basis for incorporating these effects into ODT is explained.

Focusing on DL specifically, the instability can be viewed from either a kinematical or a dynamical viewpoint. Kinematically, dilatation at an initially planar flame surface must produce streamwise acceleration on one or both sides of the flame. The equations of motion allow preservation of planar symmetry, but this mode of response to the acceleration is unstable with respect to the development of transverse spatial structure. This instability mechanism cannot be captured on a streamwise-oriented ODT domain. The failure of ODT to capture the instability kinematically does not violate any conservation laws because, as noted, the governing equations admit a solution, albeit unstable, in which the DL mechanism does not occur. Thus, the absence of this mechanism in ODT does not indicate any internal model inconsistency. Nevertheless, to achieve the best possible model representation of turbulent combustion, it is desirable to incorporate a physically sound treatment of the DL instability.

This is done by adopting a dynamical viewpoint. Consider a fluid parcel that is accelerated by flame dilatation. According to Einstein's equivalence principle [40], acceleration due to this or any other mechanism is formally equivalent 
to a gravitational body force. Accordingly, the response of the fluid parcel to acceleration can be analyzed by supposing instead that it is subject to the corresponding gravitational field. If there is density variation within the fluid parcel in the direction aligned with gravity, then density increase in the direction opposite to the orientation of the gravity vector implies gravitational instability. (This is not the only possible unstable configuration in $3 \mathrm{D}$, but it is the unstable configuration that can occur in ODT.) This is not a novel fluid-dynamical concept, but simply a formal statement of the conceptual analogy between the DL instability (or dilatational effects more generally) and the Rayleigh-Taylor (RT) instability.

In this context, ODT provides a dynamical pathway to represent the instability. The likelihood of a prospective eddy event is based on 'available energy,' a construct that incorporates the net change of gravitational potential energy caused by the eddy (here assuming buoyant stratified flow). The DL analog of this potential-energy contribution is shown in Eq. (16). Expressed as energy per unit mass, the available energy is combined with the eddy size to obtain an inverse time scale interpreted as an eddy rate that determines the eddy likelihood during a given time interval. In this manner, the ODT DL contribution can increase the intensity of a turbulent flow or initiate turbulence in a non-turbulent flow.

The need for this approach is related to the instantaneous nature of an ODT eddy. The corresponding fluid parcel in a 3D physical flow executes an eddy motion during some finite time interval. During this time interval, it is subject to any accelerations generated by the surrounding flow field and its internal structure can respond accordingly. There is neither a need nor a justification for decomposing a 3D finite-duration eddy into its evolution in a fixed reference frame and acceleration by a background flow because the equations of motion fully describe the time advancement of the entire flow domain. In contrast, an ODT eddy is instantaneous and therefore is not subject to displacement by dilatation-induced acceleration, which is a continuous-in-time process in ODT [22]. Therefore ODT does not automatically provide a mechanism for eddies 
to be influenced by dilatational flow, hence the introduction of the DL model in Sec. 2.3.3 and the possible future introduction of other such treatments. The underlying concept is that the effect of dilatation on ODT is modeled as reference-frame acceleration of ODT eddies. Importantly, this concept is applied on a cell-by-cell basis within the range of the eddy, as indicated by the spatial variation of the acceleration within the integrand of in Eq. (16), because dilatation within the eddy can cause the flow acceleration to vary within the eddy.

Though the equivalence principle is a useful basis for modeling acceleration effects in ODT, it is not fully consistent with the ODT representation of the conservation laws. In a gravitational field, the eddy-induced change of gravitational potential energy implies an equal-and-opposite change of kinetic energy, where the latter is implemented during an ODT eddy event. This conserves total energy based on an inexhaustible reservoir of gravitational potential energy. In contrast, the notional 'dilatational potential energy' change implied by Eq. (16) does not correspond to any physically existing inexhaustible potential energy source. This reflects the fact that reference-frame acceleration in ODT is based on an ad hoc partition of flow time advancement into an eddy and a background flow, where the acceleration associated with the latter does not correspond to any external mechanism that accelerates the entire system. Therefore, the notional potential energy change is not based on an external energy source analogous to the gravitational potential energy reservoir. The practical consequence is that energy conservation requires that no kinetic energy change corresponding to the potential energy change should be applied. Therefore the DL treatment affects only eddy likelihoods in a manner that is internally consistent within ODT but is not in exact conformance with the equivalence principle.

Triplet mapping during an eddy event represents a non-uniform motion, implying acceleration. Therefore it might seem that the effects of this implied acceleration should be modeled similarly in order for the treatment of acceleration to be fully consistent, but this is not done. The justification of this choice is based on the physical meaning of eddy events in ODT. 
Physical flow advancement is continuous in time and corresponds phenomenologically to an 'eddies-within-eddies' picture in inertial-range turbulence. Instantaneous ODT eddy events cannot capture this concurrent eddies-withineddies behavior. Instead, the eddies within this hierarchy must be implemented rather on the viewpoint that each eddy is a localized mode of the flow representing motion at a particular scale, as in the eddies-within-eddies interpretation of the ODT eddy-event sequence. ODT eddy events are analogous to wavelets in this respect, except that wavelets provide a decomposition of spatial structure 810 this viewpoint, modeling that assumes the triplet map to be a literal represen- 
tation of a particular flow structure rather than an abstract representation of compressional and folding effects is not well justified. 


\section{References}

815

[1] T. Poinsot, D. Veynante, Theoretical and Numerical Combustion, 3rd Edition, T. Poinsot and D. Veynante, Toulouse, 2011.

[2] S. B. Pope, Small scales, many species and the manifold challenges of turbulent combustion, Proc. Combust. Inst. 32 (2013) 1-31.

[3] A. R. Kerstein, One-dimensional turbulence: Model formulation and ap-

[9] T. Echekki, A. R. Kerstein, T. D. Dreeben, One-dimensional turbulence simulation of turbulent jet diffusion flames: Model formulation and illustrative applications, Combust. Flame 125 (2001) 1083-1105.

[10] B. Ranganath, T. Echekki, An ODT-based closure model in non-premixed 840 plication to homogeneous turbulence, shear flows, and buoyant stratified flows, J. Fluid Mech. 392 (1999) 277-334.

[4] Wm. T. Ashurst, A. R. Kerstein, One-dimensional turbulence: Variabledensity formulation and application to mixing layers, Phys. Fluids 17 (2005) 25107.

[5] A. R. Kerstein, Wm. T. Ashurst, S. Wunsch, V. Nilsen, One-dimensional tubulence: Vector formulation and application to free shear flows, J. Fluid Mech. 447 (2001) 85-109.

[6] R. C. Schmidt, A. R. Kerstein, S. Wunsch, V. Nilsen, Near-wall LES closure based on one-dimensional turbulence modeling, J. Comp. Phys. 186 (2003)

[7] R. C. Schmidt, A. R. Kerstein, R. J. McDermott, ODT: A multi-scale model for 3D turbulent flow based on one-dimensional turbulence modeling, Comput. Methods Appl. Mech. Eng. 199 (2010) 865-880.

[8] S. Wunsch, A. R. Kerstein, A stochastic model for high-Rayleigh-number convection, J. Fluid Mech. 528 (2005) 173-205.

combustion, Prog. Comput. Fluid Dynam. 6 (2006) 409-418. 
[11] J. C. Hewson, A. R. Kerstein, Local extinction and reignition in nonpremixed turbulent $\mathrm{CO} / \mathrm{H}_{2} / \mathrm{N}_{2}$ jet flames, Combust. Sci. Technol. 174 (2002) 35-66.

[12] J. C. Hewson, A. R. Kerstein, Stochastic simulation of transport and chemical kinetics in turbulent $\mathrm{CO} / \mathrm{H}_{2} / \mathrm{N}_{2}$ flames, Combust. Theory Model. 5 (2001) 669-697.

[13] D. O. Lignell, D. S. Rappleye, One-dimensional turbulence simulation of flame extinction and reignition in planar ethylene jet flames, Combust. Flame 159 (2012) 2930-2943.

[14] N. Punati, J. C. Sutherland, A. R. Kerstein, E. R. Hawkes, J. H. Chen, An evaluation of the one-dimensional turbulence model: Comparison with direct numerical simulations of $\mathrm{CO} / \mathrm{H}_{2}$ jets with extinction and reignition, Proc. Combust. Inst. 33 (2011) 1515-1522.

[15] B. Ranganath, T. Echekki, One-dimensional turbulence-based closure with extinction and reignition, Combust. Flame 154 (1981) 23-46.

[16] S. Cao, T. Echekki, A low-dimensional stochastic closure model for combustion large-eddy simulation, J. Turbul 9 (2008) 1-35.

[17] C. Glawe, F. T. Schulz, E. D. Gonzalez-Juez, H. Schmidt, A. R. Kerstein, ODTLES simulations of turbulent flows through heated channels and ducts, in: 8th Symposium on Turbulence and Shear Flow Phenomena, Poitiers, France, August 28 - 30 2013, pp. 1-6.

[18] E. D. Gonzalez-Juez, R. C. Schmidt, A. R. Kerstein, ODTLES simulation of wall-bounded turbulent flows, Phys. of Fluids 23 (2011) 125102.

[19] B. Coriton, J. H. Frank, A. Gomez, Effects of strain rate, turbulence, reacant stoichiometry and heat losses on the interaction of turbulent premixed flames with stoichiometric counterflowing combustion products, Combust. Flame 160 (2013) 2442-2456. 
[20] A. R. Kerstein, S. Wunsch, Simulation of a stably stratified atmospheric boundary layer using one-dimensional turbulence, Bound.-Lay. Meteorol. 118 (2006) 325-356.

[21] E. D. Gonzalez-Juez, A. R. Kerstein, D. O. Lignell, Fluxes across doublediffusive interfaces: A one-dimensional-turbulence study, J. Fluid Mech. 677 (2011) 218-254.

[22] D. O. Lignell, A. R. Kerstein, G. Sun, E. E. Monson, Mesh adaption for efficient multiscale implementation of one-dimensional turbulence, Theor. Comput. Fluid Dyn. 27 (2013) 273-295.

[23] A. C. Hindmarsh, SUNDIALS: Suite of nonlinear and differential/algebraic equation solvers, Tech. Rep. UCRL-JRNL-200037, Lawrence Livermore National Laboratory (2004).

880 [24] D. Goodwin, CANTERA: Object-oriented software for reacting flows. http://www.cantera.org.

[25] J. Li, Z. Zhao, A. Kazakov, F. L. Dryer, An updated comprehensive kinetic model of hydrogen combustion, Int. J. Chem. Kinet. 36 (2004) 566-575.

[26] Wm. T. Ashurst, A. R. Kerstein, Erratum: "One-dimensional turbulence: Variable-density formulation and application to mixing layers", Phys. Fluids 21 (2009) 119901.

[27] E. D. Gonzalez-Juez, A. R. Kerstein, D. O. Lignell, Reactive RayleighTaylor turbulent mixing: A one-dimensional-turbulence study, Geophys. Astrophys. Fluid Dyn. (2012) 1-20.

[28] Z. Jozefik, A. R. Kerstein, H. Schmidt, Towards a compressible reactive multiscale approach based on One-Dimensional Turbulence, in: Papers Contributed to the Conference "Active Flow and Combustion Control 2014", Berlin, Germany, Springer series Notes on Numerical Fluid Mechanics and Multidisciplinary Design, September 10 - 122014. 
[29] E. I. Monson, D. O. Lignell, C. Werver, R. S. Hintze, M. A. Finney, Z. Jozefik, A. R. Kerstein, Simulation of ethylene wall fires using the spatiallyevolving one-dimensional turbulence model, in: Fire Technology, Springer, 2014 , in press.

[30] S. Lyra, H. Kolla, B. Coriton, J. H. Frank, J. H. Chen, Counterflow $\mathrm{H}_{2} /$ air premixed flames under intense turbulence and strain, submitted to Flow Turb. Combust.

[31] T. Passot, A. Pouquet, Numerical simulation of compressible homogeneous flows in the turbulent regime, J. Fluid Mech 118 (1987) 441-466.

[32] C. S. Yoo, J. H. Chen, R. Sankaran, Direct numerical simulation of a turbulent lifted hydrogen/air jet flame in heated coflow., In Computational Combustion 2007 ECCOMAS Thematic Conference.

[33] R. S. Rogallo, Numerical experiments in homogeneous turbulence, NASA Technical Memorandum 81315, NASA Ames Research Center, CA, USA.

[34] C. K. Law, C. J. Sung, Structure, aerodynamics, and geometry of premixed flamelets, Prog. Energy Combust. Sci. 26 (2000) 459-505.

[35] F. E. Fendell, Ignition and extinction in combustion of initially unmixed reactants, J. Fluid Mech. 21 (1965) 281-303.

[36] A. Liñán, The asymptotic structure of counterflow diffusion flames for large activation energies, Acta Astronautica 1 (1974) 1007-1039.

[37] A. E. Lutz, R. J. Kee, J. F. Grcar, F. M. Rupley, OPPDIF: A Fortran program for computing opposed-flow diffusion flames, Sandia National Laboratories Report 96-8243.

[38] L. K. Su, N. T. Clemens, Measurements of the three-dimensional scalar dissipation rate field in gas phase planar turbulent jets, AIAA 97-0074, 
[39] R. W. Bilger, S. H. Starner, R. J. Kee, On reduced mechanisms for methane-air combustion in nonpremixed flames, Combust. Flame 80 (1990) $135-149$.

[40] A. Einstein, Relativity: The Special and General Theory (Translation 1920), New York: H. Holt and Company. 